* Trabalho apresentado no I Congresso Brasileiro de Epidemiologia. Campinas, Abrasco/U nicamp, 1990.

* Doutoranda em Salude Pablica (DMP/UFBA); Prof. Assis tente do Instituto de Medicina Social (Uerj); Coordenadora do Nícleo de Estudos Mulher $e$ Saúde (DMP/UF BA).

* Especialista em Medicina Social (DMP/UFBA); Pesquisadora do Nacleo de Estudos Mulher $e$ Safide (DMP/UFBA).

****Graduanda em Medicina na Faculdade de Medicina (UF BA); Estagiária em Pesquisa no Ná cleo de Estudos Mulher e Salude (DMP/UFBA).

**** Mestranda em Salude Co munitária (DMP/UFBA); Pes quisadora do Nacleo de Estudos Mulher e Salide (DMP/UFBA).

\section{Mortalidade Feminina no Brasil: Sexo Frágil ou Sexo Forte?*}

\author{
Estela Maria Leão de Aquino** \\ Greice Maria Menezes*** \\ Marúcia B. E. Amoedo**** \\ Leticia C. C. Nobre $e^{* * * * *}$
}

A população feminina brasileira tem chegado à velhice de maneira mais significativa que a masculina. Este fenômeno tem sido também observado em patses industrializados centrais onde é freqüente a sobremortalidade masculina. A análise dos diferenciais por causas espectficas pode ajudar a compreender os determinantes do padrão brasileiro e a antecipar algumas tendências futuras, especialmente tendo-se em conta as profundas mudanças no papel social da mulher em nossa realidade. Assim, foi feito estudo de mortalidade em dez capitais brasileiras, em 1985, com taxas geral e espectficas por cinco principais grupos de causas segundo sexo e padronizadas por idade pelo método direto. Como medida dos diferenciais, usou-se razóes e diferenças entre os sexos. Os resultados revelam que o perfil regional relaciona-se ao padrão de urbanizaçâo e industrialização, com os maiores diferenciais por sexo nas capitais mais desenvolvidas do pats. A sobremortalidade masculina explica-se basicamente pela magnitude das causas externas e das doenças do aparelho circulatorio, sendo peculiar a especial importância das mortes violentas, que são as principais responsáveis pelos diferenciais por sexo. Acredita-se que a atual tendência venha a se manter, embora a longo prazo as diferenças entre os sexos possam se reduzir. De qualquer modo, o prolongamento da vida, sem adequadas condiçōes de subsistência, nāo parece estar significando exatamente uma vantagem para a mulher brasileira.

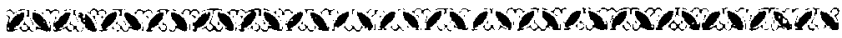

\section{INTRODUÇÃO}

Apesar de ainda ser predominantemente jovem, a população brasileira tem envelhecido de modo muito rápido, sem que isso signifique uma real melhoria das condições de vida de sua grande maioria (Martine \& Camargo, 1984; Ramos et alii, 1987; Saad \& Camargo, 1989). 
Com provável caráter definitivo, especialmente pela irreversibilidade da esterilização do amplos contingentes de mulheres em idades precoces, o envelhecimento não tem sido uniforme entre os sexos. $O$ aumento da expectativa de vida ao nascer que, entre 1920 e 1980, situou-se ém tomo de 30 anos para ambos os sexos, foi mais significativo para as mulheres. As diferenças são presentes em todas as regiōes do pais e, em 1980, as mulheres apresentavam vantagens sobre os homens que variavam de 5,8 anos no Nordeste a 7,1 anos no Sul (A Mulher brasileira, 1986).

Essa tendência é consistente com o descrito na literatura a respeito da ocorrência freqüente do fenômeno de subremortalidade masculina em países industrializados centrais, desde o início deste século. Nessas sociedades, especialmente após o controle da mortalidade materna, os homens tenderam a morrer mais precocemente que as mulheres, apresentando maiores taxas de mortalidade específicas por idade para a maioria das causas de morte (Verbrugge, 1982; Wingard et alii, 1983; Wingard, 1982).

Durante muito tempo, estas diferenças foram explicadas essencialmente por diferenças biológicas entre os sexos, tais como maior imunidade das mulheres para doenças infecciosas, maior proteção hormonal feminina para as doenças coronarianas etc (Waldron, 1976). Ainda hoje, no âmbito da Epidemiologia, embora a categoria sexo seja contemplada na grande maioria dos estudos, esta aparece na sua dimensấo estritamente biológica e as diferenças, de um modo geral, tendem a ser naturalizadas.

Assumimos como pressuposto teórico que o sexo dos indivíduos é construído, socialmente, de modo diferenciado entre classes, grupos culturais e étnicos, e como tal precisa passar a ser explorado para melhor compreensão dos fenômenos.

O presente estudo teve por objetivo identificar as principais características do padrăo de mortalidade brasileiro quanto aos deferenciais por sexo em diferentes grupos de idade e segundo grupos de causas específicas, de modo a obter indicaçöes relativas aos determinantes do padrão de envelhecimento nacional e a possiveis tendências futuras, especialmente considerando as profundas mudanças que vêm ocorrendo na inserção da mulher em nossa sociedade.

\section{M.ATERIAL E MÉTODO}

$O$ estudo foi realizado a partir de dados consolidados pelo Ministério da Saúde sobre b́bitos ocorridos em 1985 em dez capitais brasileiras. Foram construídos coeficientes de mortalidade geral e específica por cinco principais grupos de causas segundo sexo, padronizados por idade pelo método direto (Kirkwood, 1988). 
Como medidas dos diferenciais, foram calculadas razōes de mortalidade entre os sexos, ou índices de sobremortalidade masculina (ISM) por idade. Para a análise da contribuição dos grupos de causas na sobremortalidade masculina, foram calculadas diferenças absolutas e relativas (\%) entre os coeficientes específicos por sexo.

Os dados foram processados em microcomputador PC-XT, utilizando-se o programa Lótus. Para representação gráfica, adotou-se o Harvard Graphics.

\section{RESULTADOS}

Em 1985, nas dez capitais selecionadas, o grupo de doenças do aparelho circulatório foi de longe o principal responsável pelos óbitos (gráficos I e II). As causas externas foram o segundo grupo mais importante em pessoas do sexo masculino, exceto em Salvador e em Porto Alegre, onde foram ultrapassadas, respectivamente, pelas doenças infecciosas e parasitárias e pelas neoplasias. Em mulheres foi o câncer que apareceu em segundo lugar de importância, à exceçấo de Belém, de Fortaleza e de Salvador. Nestas capitais, as doenças infecciosas e parasitárias foram mais relevantes como causas de morte.

\section{GRÅFICO I}

Mortalidade* por 5 Grandes Grupos de Causas em Mulheres em 10 Capitais Brasileiras - 1985

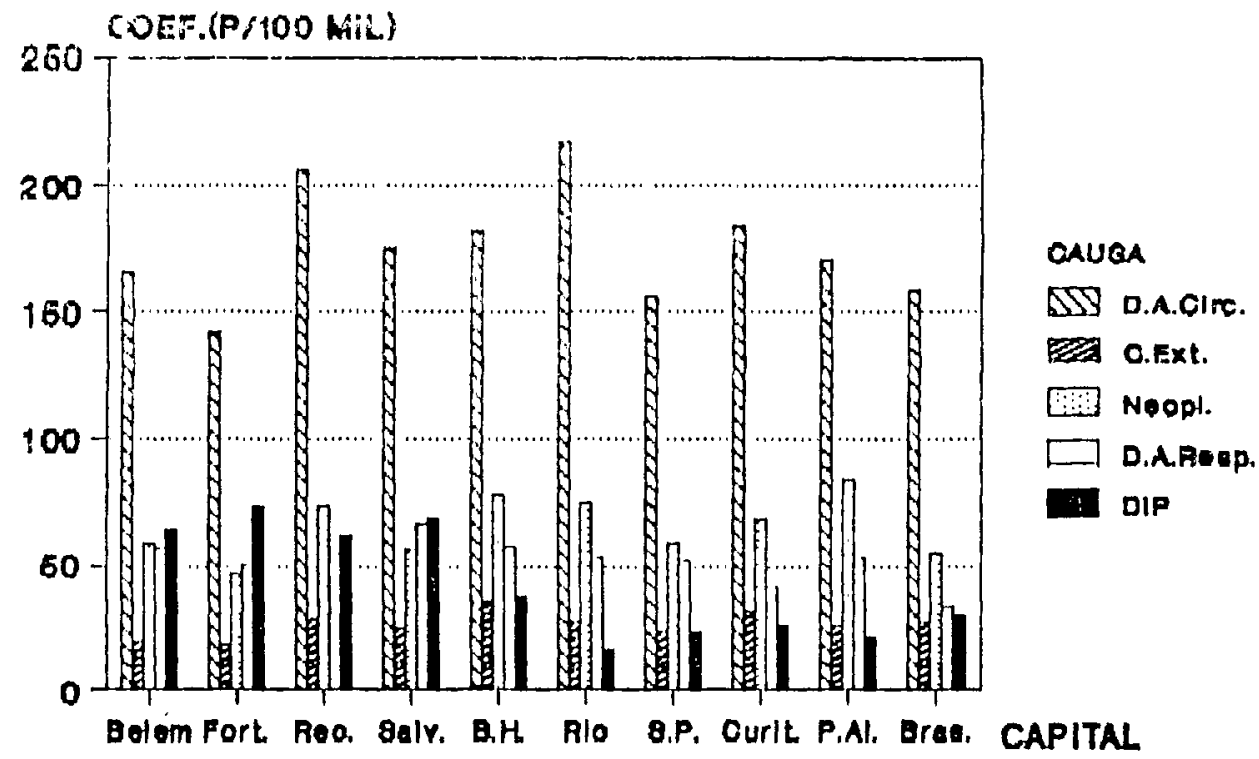

Fonte: M.S., Estat. Mortalidade, 1985.

"coef. padronizados pela pop. bras. de 1980. 


\section{GRÁFICO $\amalg$}

Mortalidade* por 5 Grandes Grupos de Causas em Homens em 10 Capitais Brasileiras - 1985

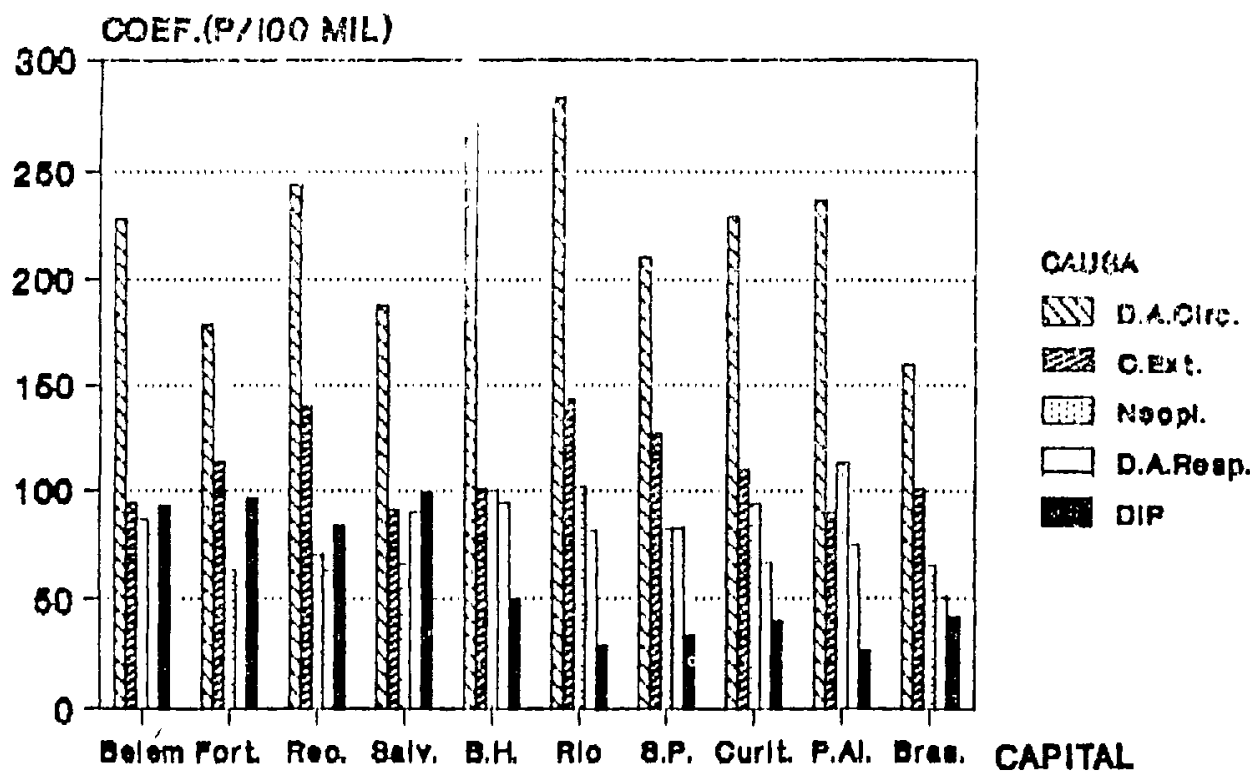

Fonte: M.S., Estat. Mortalidade, 1985.

"coef. padronizados pela pop. bras. de 1980.

A sobremortalidade masculina foi constante em todas as capitais (gráfico III): os menores diferenciais estavam no Nordeste e os maiores no Rio de Janeiro, em São Paulo e em Brasillia.

Entre os principais grupos de causas, os diferenciais assumiram valores extremamente altos nas causas externas, quando o risco de morrer masculino foi de três a mais de cinco vezes o do sexo feminino (gráfico IV). Isso fica evidente na análise da contribuição de grupos de causas definidas para sobremortalidade masculina. Em todas as capitais, as causas externas "explicaram" cerca de 30 a $40 \%$ dos diferenciais, sendo o principal grupo, exceto em Belo Horizonte e em Porto Alegre (gráficoV). Nessas duas capitais foram superadas em importância relativa pelas doenças do aparelho circulatório que, embora apresentando menores diferenciais, assumiram relevância pela sua magnitude como causas de morte. Em todas as capitais, esses dois grandes grupos de causas responderam por metade ou mais do "excesso" de mortes masculinas.

Cadernos de Saude Páblica, RJ, 7 (2): 174-189, abr/jun, 1991 


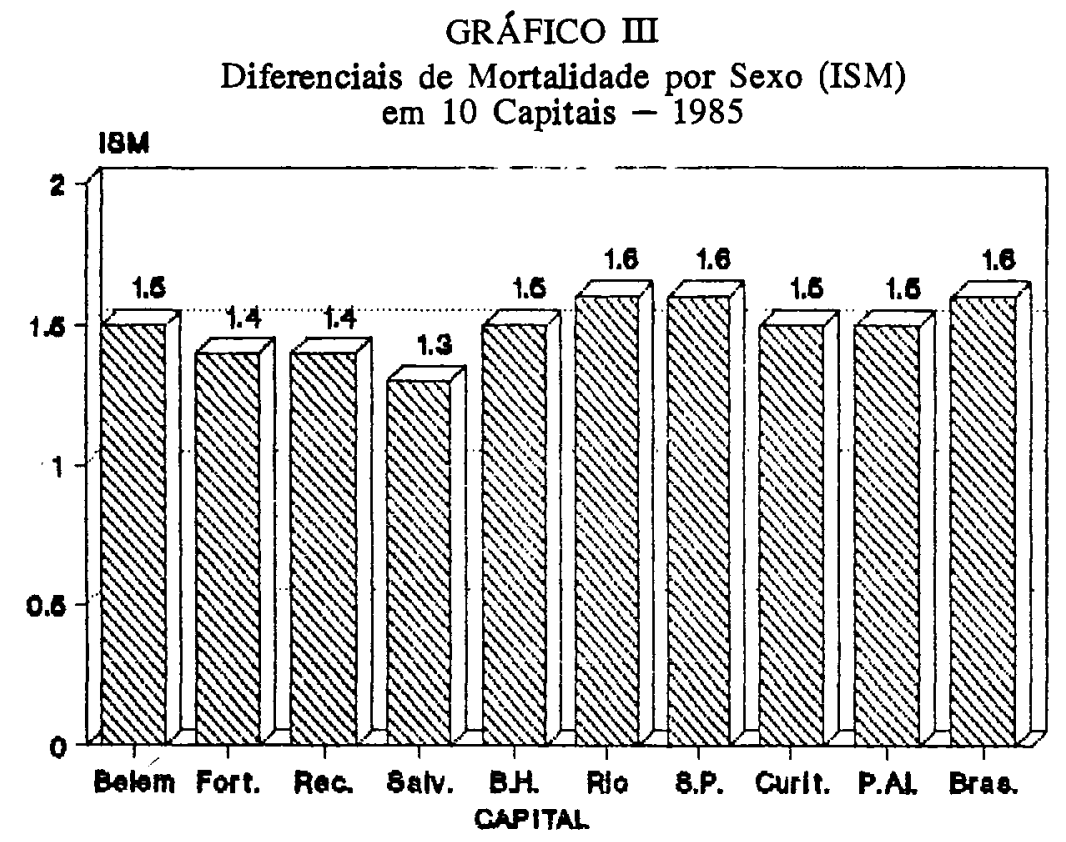

Fonte: M.S., Estat. Mortalidade, 1985.

\section{GRÁFICO IV}

Diferenciais de Mortalidade por Sexo (ISM) de

5 Grupos de Causas em 10 Capitais - 1985

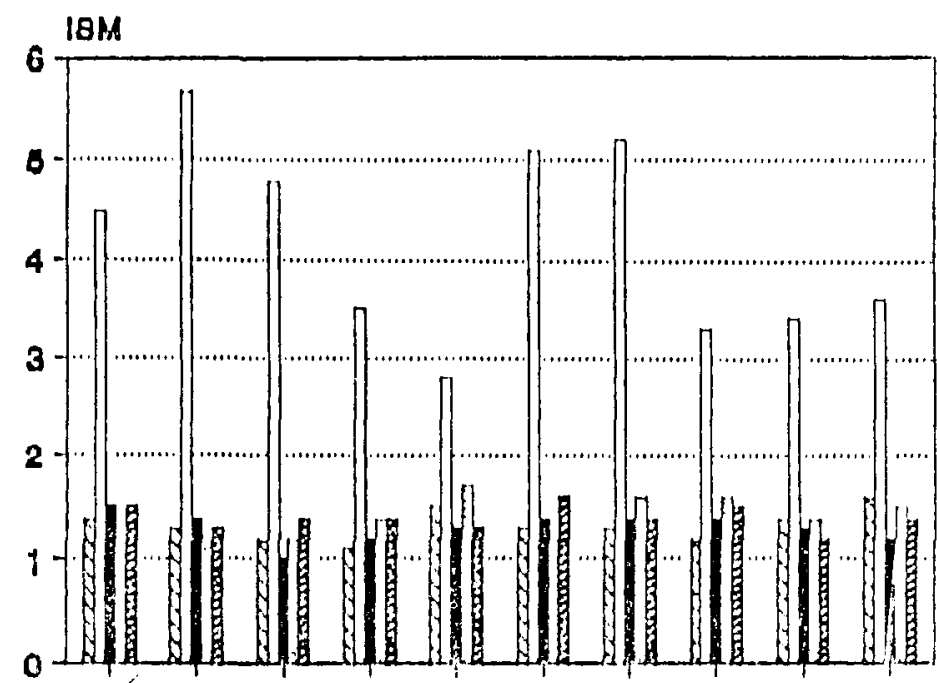

CAUEA

DS D.A.CIre.

$\square$ C.Ext.

r. Neod.

D.A.ASap.

DIP

Bolón rort. Roa gals. B.H NID g.P. Curlt P.Al, BIBa, CAPITAL

Fonte: M.S., Estat. Mortalidade, 1985. 


\section{GRÁFICO V}

Contribuição Percentual de 5 Grupos de Causas nos Diferenciais de Mortalidade por Sexo em 10 Capitais - 1985

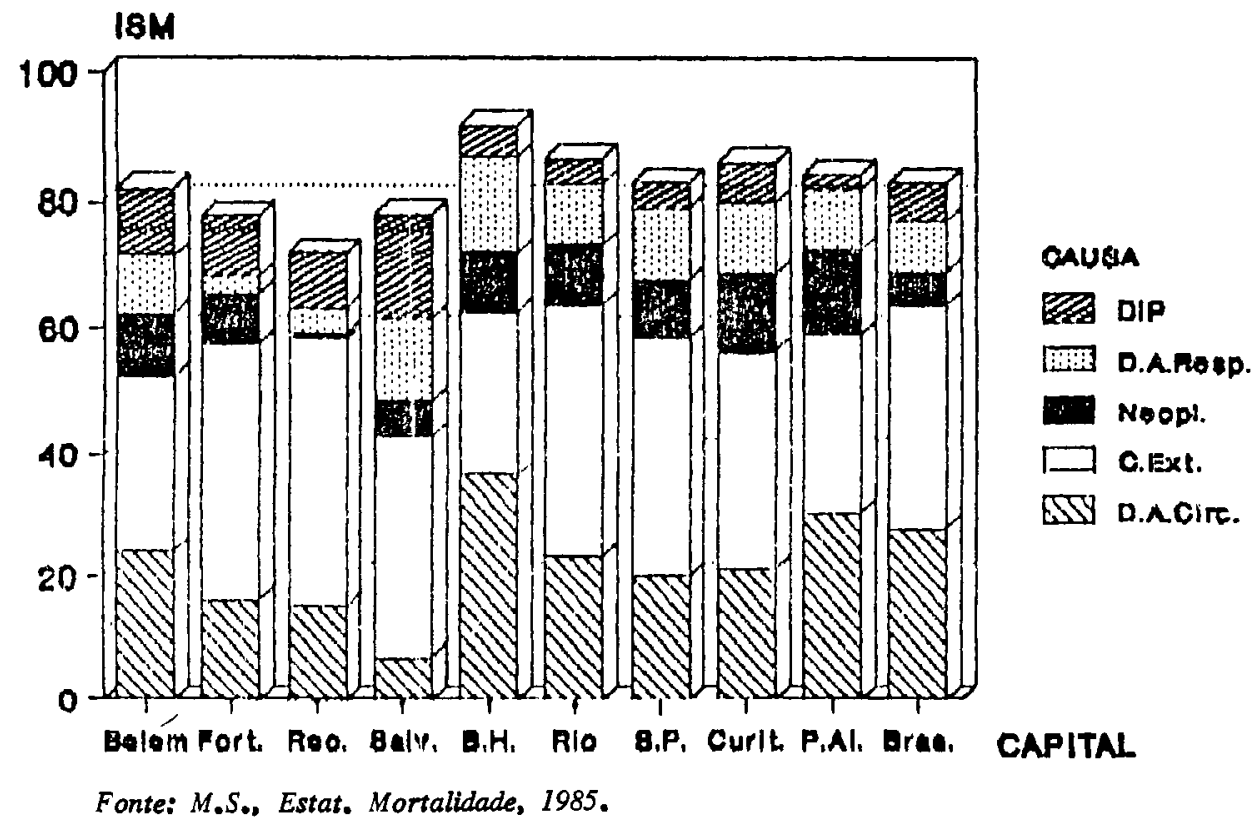

As maiores diferenças entre os sexos situaram-se nas faixas de 15 a 19 anos e de 20 a 29 anos (gráficos VI a VIII). Embora, de um modo geral, identifique-se uma curva unimodal com o pico localizado em torno dessas faixas etárias, grosseiramente podem ser observados três padrōes.

Um primeiro tipo, onde a curva apresenta um pico bem acentuado de 20 a 29 anos (em São Paulo, de 15 a 19 anos), quando o risco de morrer entre os homens é de três a cinco vezes maior que o das mulheres (gráficos VI). Este padrão foi observado em São Paulo, em Recife, em Porto Alegre, em Fortaleza e em Brasília. Um segundo padräo, semelhante ao primeiro, apresenta um platô entre 15 e 29 anos, onde encontram-se riscos de morrer em tomo de três vezes naaiores nos homens (gráfico VII). Este padrão foi observado no Rio de Janeiro e em Curitiba. Por último, Salvador, Belo Horizonte e, especialmente, Belém apresentam curva mais suave, embora em adultos jovens a sobremortalidade ainda seja da ordem de 2 a 2,5 (gráfico VIII). De qualquer forma, nos três con-

Cadernos de Saúde Pública, RJ, 7 (2): 174-189, abr/jun, 1991 
juntos os diferenciais são praticamente nulos em crianças e tendem a se reduzir nas idades mais altas, chegando quase a desaparecer entre os mais velhos.

GRÁFICO VI

Diferenciais de Mortalidade por Sexo e Idade em Fortaleza, Recife, Brastlia, S. Paulo e Porto Alegre - 1985

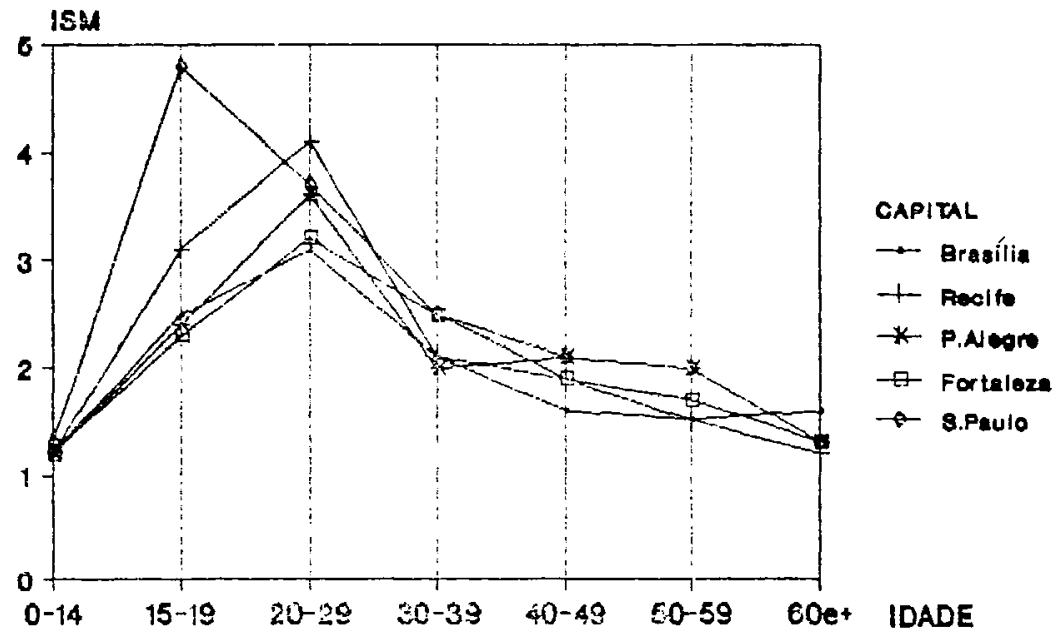

Fonte: M.S., Estattsticas de Salde, 1985.

\section{GRÁFICO VII}

Diferenciais de Mortalidade por Sexo e Idade em Curitiba e Rio de Janeiro - 1985

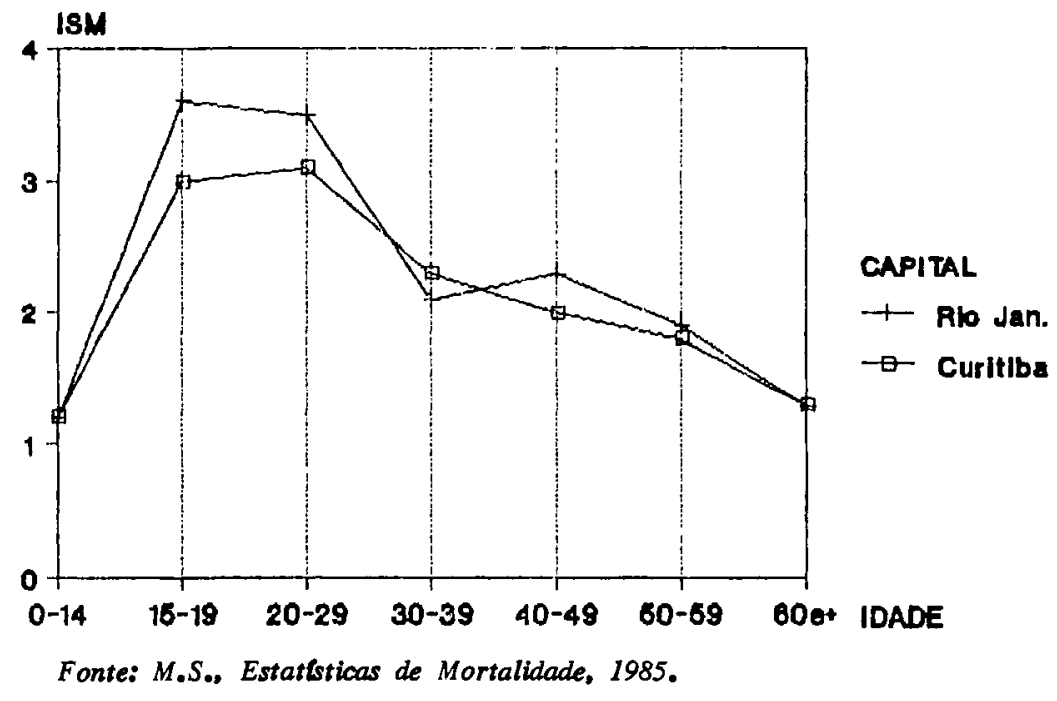




\section{GRÁFICO VIII}

Diferenciais de Mortalidade por Sexo e Idade em Belém, Salvador e Belo Horizonte - 1985

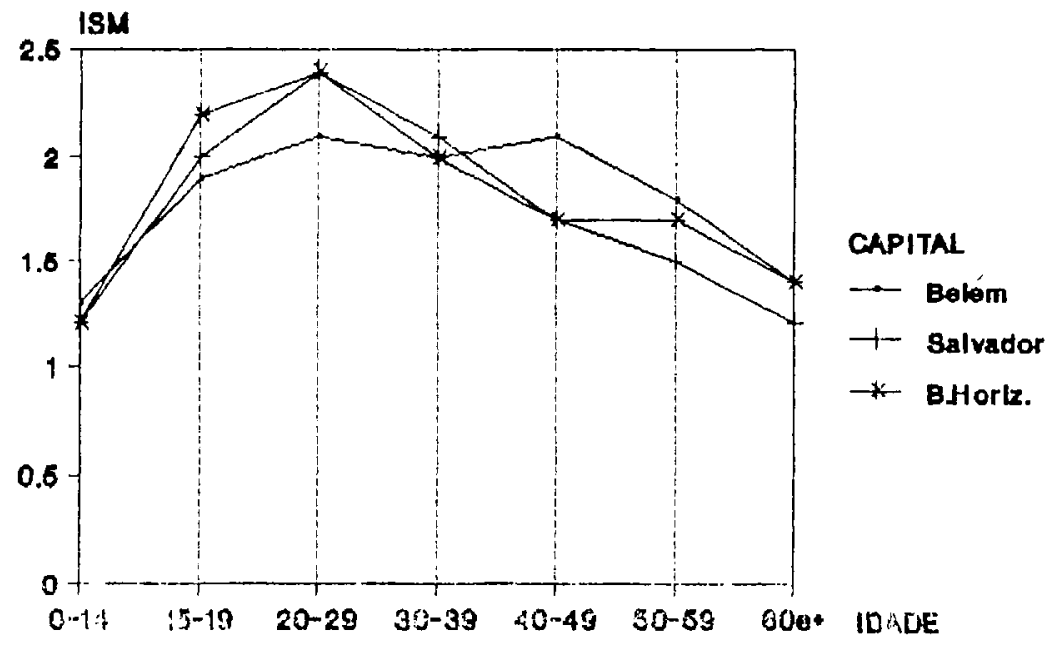

Fonte: M.S., Estatisticas de Mortalidade, 1985.

\section{DISCUSSÃO}

O padrão de mortalidade observado, com predomínio acentuado das doenças do aparelho circulatório como causas de morte em ambos os sexos, vem se acentuando desde a década de 60, quando este grupo superou as doenças infecciosas e parasitárias e passou a ser o principal responsável pelos óbitos do país (Mortalidade nas capitais, 1984). Esta tendência ao aumento da relevância de doenças crônico-degenerativas e das violências como causas de morte vem se aprofundando em todo o pafs. Contudo, nunca é demais ressaltar a coexistência de mortes por doenças do atraso e da modernidade, especialmente no Norte e no Nordeste. As doenças infecto-parasitárias como causas de morte não têm qualquer expressão em países industrializados centrais desde o início deste século (Lewis \& Lewis, 1977; Verbrugge, 1980).

A constatação da existência dos maiores diferenciais por sexo nas capitais mais desenvolvidas é compatível com a tendência história de aumento da sobremortalidade masculina concomitante ao processo de urbanização e industrialização do país. Também é consistente com a literatura, onde se observam maiores indices de sobremortalidade masculina nos países desen- 
volvidos que nos subdesenvolvidos (Wingard, 1984). Nestes últimos, os diferenciais são mais variados, sendo mencionado o exemplo da India, onde há um padräo inverso com vantagem de três anos para os homens na expectativa de vida ao nascer.

A contribuição de causas como as doenças cardiovasculares e de causas externas para os diferenciais por sexo é também descrita em outros estudos. $O$ que $\varepsilon$ peculiar à realidade brasileira diz respeito à enorme contribuição das causas violentas para os diferenciais por sexo, não apenas pelo elevadíssimo risco masculino de morrer por estas causas, mas também pela grande magnitude que estas assumem no Brasil. Embora sejam relatadas variaçōes na magnitude dos diferenciais entre os sexos na mortalidade por doenças cardiovasculares (Waldron, 1976), estudos norte-americanos (Nathanson, 1977; Wingard, 1984; Waldron, 1976), apontam este grupo de doenças (especialmente as do coração) como as principais responsáveis pelo "excesso" de mortes masculinas. Os neoplasmas, que as seguiriam em ordem de importância, nâo têm grande expressão em nossa realidade como contribuintes para os diferenciais, provavelmente pela relevância de neoplasias como as do colo uterino, sob controle em países industrializados centrais, que ocasionam al tas taxas de mortalidade femininas (Faerstein, Aquino \& Ribeiro, 1989) e, conseqüentemente, diminuem as diferenças entre os sexos.

Os padrões etários de curva unimodal com pico em idades jovens são bastante semelhantes aos observados em países do sul e do leste europeu (Lopez, s.d.). Certamente a importância inquestionável das causas violentas de morte vai explicar a ocorrência dos maiores diferenciais em torno dos 20 anos. Em países europeus, nos EUA e no Canadá, é relatada a existência de um segundo pico em torno dos 60 anos (Nathanson, 1977; Wingard, 1984; Lopez, s.d.), o que não ocorre nas capitais brasileiras estudadas, onde se observam valores quase nulos nos extremos das curvas etárias.

$\mathrm{Na}$ literatura revista, possíveis explicações para os diferenciais de mortalidade entre os sexos têm envolvido fatores biológicos, sociais e comportamentais.

A favor dos primeiros falariam as evidências de maiores taxas de mortalidade entre fetos do sexo masculino e entre meninos no primeiro ano de vida, o que seria também observado em outras espécies. Os estudos, porém, apresentam resultados contraditórios, tanto em relação ao papel de fatores genéticos quanto ao de hormônios sexuais (Waldron, 1976).

Dessa forma, ainda que nenhum autor exclua a contribuição dos fatores biológicos, os trabalhos com ênfase nos fatores sociais e comportamentais vêm pro- 
gressivamente ganhando mais espaço. A grande maioria, utilizando teorias de estilo de vida, faz inferências sobre determinantes das diferenças na mortalidade entre os sexos, a partir da análise da contribuição de causas especificas de morte.

Os principais determinantes da sobremortalidade masculina apontadós dizem respeito às diferenças no consumo de álcool e de tabaco, fatores de risco para os neoplasmas e para as doenças cardiovasculares; às diferenças na exposição a fatores de riscos do trabalho; a hábitos masculinos mais perigosos, como a direção de veículos e o uso de armas, e a comportamentos masculinos mais agressivos e mais competitivos, contribuindo para as violências e para um maior stress cotidiano.

Mudanças no papel das mulheres em diferentes sociedades ocidentais têm despertado o interesse sobre o impacto possível nos diferenciais de adoecimento e de morte entre os sexos. Ao que parece, as chamadas "vantagens" femininas quanto ao tempo de sobrevida poderiam estar se reduzindo em decorrência dessas mudanças. Nos EUA, por exemplo, a partir da década de 70 , observam-se três tipos de modificações de tendências (Verbrugge, 1980):

- para muitas causas, como as doenças cerebrovasculares, os acidentes de veículos e outros acidentes, a arteriosclerose e as anomalias congênitas, houve uma estabilização nos diferenciais;

- para outras causas, como as doenças pulmonares (enfisema, asma, bronquite), os homicídios, a úlcera péptica e as doenças da tenra infância, os diferenciais diminuíram com "erosão da vantagem feminina";

- para todas as demais causas principais, como as doenças do coração, os neoplasmas, a cirrose do figado, os suicídios, as pneumonias, as nefrites e as nefroses, o incremento observado tem sido mais lento do que em anos anteriores.

No Brasil, o prapel da mulher tem mudado muito, especialmente nas últimas duas décadas. Isso se expressa na crescente participação feminina como força de trabalho e como chefe de família, nas mudanças de comportamento quanto ao controle da reprodução e na maior organizaçăo política, através de movimentos específicos de mulheres.

O impacto dessas mudanças sobre a saúde ainda é pouco conhecido. É possível que as especificidades do processo em nossa realidade estejam conformando unı padrão peculiar, com características bastante diversas das observadas em outras sociedades.

Em primeiro lugar, a inserção profissional, embora em velocidade acentuada, ainda se dá, de modo privilegiado, em ocupaçōes tipicamente "femininas" (Medici, 1989), expondo as mulheres a fatores de risco

Cadernos de Saúde Pública, RJ, 7 (2): 174-189, abr/jun, 1991 
diferenciados dos experimentados pelos homens. Por'tanto, a entrada no mercado de trabalho não implicaria, necessariamente, uma equiparação de homens e mulheres quanto aos problemas de saúde relacionados ao trabalho. Por outro lado, essa crescente inserção profissional não tem sido acompanhada da criação de mecanismos sociais, seja através de equipamentos coletivos como creches, lavanderias etc, seja pela mudança nas relaçöes entre os sexos (extremamente marcadas pelo patriarcalismo) que as liberem de suas tarefas tradicionais nos cuidados da casa e dos filhos. Como resultado, é possível supor que os efeitos do trabalho sobre a saúde da mulher brasileira não se reproduzam necessariamente com valor benéfico como o observado em países industrializados centrais (Nathanson, 1975; Sorensen \& Verbrugge, 1987; Waldron, 1983; Wingard, 1984).

A redução acentuada do número de filhos, em todas as regiōes do país, tem sido atribuida, entre outras causas, à necessidade efetiva de controle da prole, principalmente com a progressiva incorporação da mulher ao mercado de trabalho. Essa queda da fecundidade tem se dado não apenas pela esterilização de amplos contingentes de mulheres, mas também pela utilização maciça e indiscriminada de anticoncepcionais orais, que são fatores de risco para neoplasias e para doenças cardiovasculares. Pressupōe-se inclusive que essa utilização abusiva, sem orientação médica, seja responsável, ao menos em parte, pelas altas taxas de mortalidade por doenças cerebrovasculares (Health policy, 1989), que corresponderam a 35,5\% das mortes por aquele grupo de causas ocorridas, em 1985, em mulheres adultas (Aquino, 1989).

As mudanças de comportamento têm envolvido a incorporação de hábitos, como o de fumar, com conhecidas implicaçōes para a saúde. O tabagismo está associado a doenças respiratórias (bronquite, asma e enfisema) e a doenças cardiovasculares; é sabidamente o mais importante fator de risco isolado e removivel na prevençäo do câncer, implicando o aumento de risco para neoplasias de pulmẩo, boca e faringe, laringe, esôfago, bexiga, rim e pâncreas (Faerstein, Aquino \& Ribeiro, 1989).

Com o progressivo controle do consumo de cigarros em países do primeiro mundo, as indústrias do fumo deslocaram seus investimentos para os paises periféricos, com grande ofensiva publicitária. A exemplo do que ocorrera em sociedades desenvolvidas (Ernster, 1985), essa publicidade envolveu estratégias dirigidas ao público feminino, associando fumo e "emancipação", e o lançamento de "marcas femininas". Como resultado, a prevalência de fumantes que, após décadas de rápido aumento, vem diminuindo, 
mantém-se entretanto crescente entre mulheres jovens, principalmente as mais pobres e menos escolarizadas (Health policy, 1989).

Em relação ao padrão alimentar, embora as informações no país sejam bastante desatualizadas, presume-se que, ao menos entre as camadas mais pobres, que constituem a grande maioria da população, a dieta continue deficiente em proteínas, vitaminas e fibras. Por outro lado, a prevalência de obesidade, maior entre os pobres, é particularmente alta entre mulheres com baixa escolaridade (Health policy, 1989). Este padräo teria implicaçōes no aumento do risco para doenças cardiovasculares e para neoplasias (como as de cólon e de mama, por exemplo, especialmente relevante na população feminina).

A prática de exercícios, apontada como fator de proteção para problemas cardíacos, em recente inquérito nacional, revelou-se mais freqüente entre homens $(30 \%)$ do que entre mulheres $(18 \%)$, estando diretamente relacionada ao nível de escolaridade (Health policy, 1989).

Os reflexos da combinação desses fenômenos sobre a saúde são ainda imprevisíveis, mais poder-se-ia especular com algumas hipóteses.

Os diferenciais de mortalidade por doenças crônico-degenerativas devem se manter ou mesmo aumentar em um primeiro momento. Estudos sobre a evolução das doenças do aparelho circulatório em São Paulo descrevem uma tendência ao aumento da mortalidade até meados da década de 70 (Laurenti \& Fonseca, 1977). A partir de 1976, começa a haver um declínio em ambos os sexos, principalmente das doenças isquêmicas do coração, cujo decréscimo é maior nas mulheres (Lolio et alii, 1986; Lolio \& Laurenti, 1986a). A queda da mortalidade por doenças cerebrovasculares, entretanto, \& maior entre os homens (Lolio \& Laurenti, 1986b).

Pode-se supor que, estando os acidentes vasculares cerebrais e as doenças isquêmicas do coração entre as complicaçöes mais freqüentes da hipertensão arterial, descrita como associada ao stress, mudanças na inserção social da mulher venham a ter impacto, a médio e longo prazos, sobre a mortalidade por doenças cardiovasculares, com diminuição dos diferenciais entre os sexos.

A ocorrência das neoplasias, segundo grupo mais importante na população feminina adulta, não tem sido alvo de ações efetivas de controle, que possam reduzir a alta magnitude de cânceres tipicamente femininos como os de colo de útero e de mama. Em contrapartida, é possível que o aumento da prevalência do fumo, mais acentuado entre as mulheres, ocasione uma elevaçẩo mais expressiva das neoplasias de pulmäo nesse 
sexo, levando a uma diminuição do diferencial por sexo (Faerstein, Aquino \& Ribeiro, 1989). De qualquer forma, estas mudanças devem demorar a ser sentidas, devido aos longos períodos de latência das doenças em questão.

Dentro desse panorama, um elemento novo é o aparecimento da AIDS (Síndrome da Imunodeficiência Adquirida) que, atingindo preferencialmente homossexuais masculinos, em um primeiro momento pode contribuir para o aumento dos diferenciais. Porém, há que se considerar que, no Brasil, o elevado número de homens com histórias de relaçōes bissexuais, a grande freqüência de práticas de coito anal entre casais e o controle deficiente do sangue e derivados para transfusões têm contribuído para a expansão da doença além dos clássicos grupos de risco (Health policy, 1989).

De qualquer modo, o aumento da sobremortalidade masculina por todas as causas deve se manter no curto prazo, especialmente porque um dos principais componentes para sua manutenção - o maior risco masculino de morrer por causas extemas - não deve ser afetado de modo substancial pelas mudanças recentes no papel social da mulher. É muito possível que o acirramento das contradições sociais na conjuntura recente contribua, inclusive, para perpetuação da tendência ao aumento das causas violentas de morte, que tem sido identificada em estudos regionais (A Mulher brasileira, 1986; Ortiz \& Yamazaki, 1984).

Como conseqüência, o envelhecimento populacional rápido, com especial "vantagem" para as mulheres, deve se manter no futuro mais imediato. Cabe indagar em que condiçōes esse processo tem se dado e o que significa o prolongamento da vida em nossa realidade?

A violência que se expressa através das estatísticas de mortalidade, e de modo inegável atinge prioritariamente a população masculina, corresponde à face visível de uma realidade, onde as desigualdades assumem formas menos evidentes, mas nem por isso menos perversas. O "lado submerso do iceberg" esconde um mundo de violências não-declaradas, que envolvem as discriminações de raça, o abandono de menores, a fome de milhões de trabalhadores, e particularmente a violência cotidiana contra mulheres no espaço inviolável do lar. Esta violência, que raramente ganha as páginas dos jomais e tende a ser tratada como"natural", decorre de relaçōes de gênero assimétrico, onde a mulher ocupa posição subalterna. Ganha alguma visibilidade apenas quando são criados mecanismos sociais como as delegacias de mulheres e outros organismos de apoio às vítimas. As agressões que chegam a ser denunciadas, embora em sua maioria não resultem em mortes, nem por isso causam pouco sofrimento: são 
praticadas no espaço domiciliar, por maridos, companheiros e outros familiares (Goldenberg, Medrado \& Pasternostro, 1989).

Uma outra forma de violência cotidiana diz respeito à situação da pessoa idosa no Brasil. A mulher velha, sobrevivendo mais, está mais exposta à viuvez, e menos instrumentalizada que o homem para complementar aposentadorias e pensōes miseráveis. Em uma sociedade marcada pelas desigualdades, o maior envelhecimento feminino tem significado, portanto, a combinação de pobreza, doença e solidão.

A perspectiva de agravamento das precárias condiçōes de vida pela crise econômica e pelo arrocho salarial certamente torna o horizonte ainda mais sombrio. Desse modo, não é possível supor que, em nossa perversa realidade, o prolongamento da vida, sem adequadas condiçōes de subsistência, esteja significando exatamente uma vantagem.

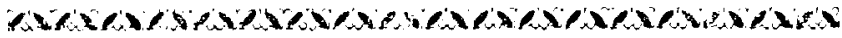

Population ageing in Brazil has been more rapid and more intense among women. This phenomenon is well described in developed countries where mortality rates are higher for men than women. In this regard, the analysis of mortality patterns by cause contributes to elucidate the determinant factors of the present situation in Brazil and provides indications of some future trends in female mortality. This is especially important due to the fact that in Brazil the social role of women has experienced great changes. This study presents data on mortality from ten capital cities in 1985, showing age-standardized overall and cause-specific mortality rates for five of the main groups of causes by sex. Ratios and differences effect estimators were used. The results revealed that regional pattern:: are associated with the urban and industrial processes with greater differences by sex in more developed regions. External causes and cardiovascular diseases are the main factors responsible for higher mortality among men with special emphasis on violent deaths. It is inferred that the present trend will be maintained, though it is possible that mortality differences by sex could decrease in the near future. The authors discuss that longer survival among women in Brazil does not reveal better life conditions. 


\section{REFERÊNCIAS BIBLIOGRÁFICAS}

A MULHER brasileira: Estatifticas de satide. Radis/Dados out. 1986; 4 (10): 1-24.

AQUINO, E.M.L. Satide da mulher no Brasil: antigas e novas necessidades. In: C.N.D.M. Quando a paciente $f$ mulher. Brasllia, C.N.D.M./MJ., 1989.

ERNSTER, V.L. Mixed messages for women: a sacial history of cigarette smoking and advertising New York State Journal of Medicine July, 1985: 335-40.

FAERSTEIN, E., AQUINO, E.M.L. \& RIBEIRO, D.C.S. Câncer na mulher: uma prioridade no Brasil? In: LABRA, E. Mulher, Salude e Sociedade no Brasil. Petrópolis, Ed. Vozes/Abrasco, 1989. p. 163-83.

GOLDENBERG, P., MEDRADO, M.A. \& PASTERNOSTRO, M.A.N. A violência contra a mulher: uma questăo de saúde. In: LABRA, E (org.) Mulher, Satide e Sociedade no Brasil. Petropolis, Ed. Vozes/Abrasco, 1989. p. 185-200.

HEALTH policy in Brazil: adjusting to new challenges. Washington, World Bank, draft of May 15, 1989.

KIRKWOOD, B.R. Essentials of Medical Statistics. London, Blackwell Scientific Publications, 1988. p. 112-4.

LAURENTI, R. \& FONSECA, L.A.M. A evoluçāo da mortalidade por doença isquêmica do coraçăo no municfpio de Săo Paulo de 1940 a 1973. Arq. Bras. Cardiol., 1977; 30 (5): 351-5.

LEWIS, C.E. \& LEWIS, M.A. The potential impact of sexual equality on health. New England Joumal of Medicine, Oct. 20, 1977; 297 (16): 863-9.

LOLIO, C.A. et alii. Decline in cardiovascular disease mortality in the city of São Paulo, Brazil, 1970 a 1983. Rev. Saúde Pabl., 1986; 20 (6): 454-64.

LOLIO, C.A. \& LAURENTI, R. Mortalidade por doença isquêmica do coraçåo no munićpio de São Paulo: evoluçăo de 1950 a 1981 e mudanças recentes na tendência. Arq. Bras. Cardiol., $1986 \mathrm{a} ; 46$ (3): 153-6.

LOLIO, C.A. \& LAURENTI, R. Tendéncia da mortalidade por doenças cerebrovasculares em adultos maiores de 20 anos de idade no município de Såo Paulo (Brasil), 1950 a 1981. Rev. Salude Pábl., 1986; 20 (5): 343-6.

LOPEZ, A.D. The Sex Differential in Mortality in Developed Countries, In: LOPEZ, D. \& RUZICKA, L.T. (Ed.) Sex Differentials in Mortality: Trends, Determinants and Consequences. Camberra, Australian Nat. Univ. Press, s.d. p. 53-120.

MARTINE, G. \& CAMARGO, L. Crescimento e distribuiçăo da população brasileira: tendências recentes. Rev. Bras. Est. Popul. Jan/dez, 1984; 1 (1): 99-144.

MÉDICI, A.C. Mulher brasileira: muito prazer. In LABRA, E. (org.) Mulher, Salide e Sociedade no Brasil. Petrópolis, Ed. Vozes/Abrasco, 1989. p. 71-117.

MORTALIDADE nas capitais brasileiras. 1930-80. Radis/Dados Ago, 1984; (7): 1-8.

Cadernos de Saúde Páblica, RJ, 7 (2): 174-189, abr/jun, 1991 
NATHANSON, C.A. Illness and the Feminine Role: Theoretical Review. Soc. Sci. Med., 1975; 9: 57-62. (47 ref.)

NATHANSON, C.A. Sex, Illness and Medical Care. A Review of Data, Theory and Method, Soc. Sci. Med., 1977; 11:13-25. (88 ref.)

ORTIZ, L.P. \& YAZAKI, L.M. Aumento do diferencial por sexo da mortalidade no Estado de Såo Paulo. Rev. Bras. Est. Popul. 1984; 1 (1/2): 145-70.

RAMOS, L.R. et alii. Envelhecimento populacional: uma realidade brasileira, Rev. Salide Pabl., 1987; 21 (3): 214-24.

SAAD, P.M. \& CAMARGO, A.B.M. O envelhecimento populacional e suas conseqüências, Säo Paulo em Perspectiva, jul/set, 1989; 3 (3):40-5.

SORENSEN, G. \& VERBRUGGE, L.M. Women, work and health. Ann. Rev. Public Health, 1987; 8: 235-51. (125 ref)

VERBRUGGE, L.M. Recent trends in sex mortality differentials in the United States. Women \& Health 1980; 5 (3): 17-37. (31 ref.)

VERBRUGGE, L.M. Sex Differentials in Health. Public Health Reports. Sep-Oct. 1982; 97 (5): 417-37.

WALDRON, I. Employment and women's health: an analysis of causal relationships. In: FEE, E. (Ed.) Women and health: the politics of sex in Medicine. Farmingdale, N.Y., Baywood Publishing Company, Inc., 1983. Policy, Politics, Health and Medicine Series n: 4, chap. 4, p. 119-38. (107 ref.).

WALDRON, I. Why do women live longer than men? Soc. Sci. Med. 1976; 10: 349-62.

WINGARD, D.L. et alii. The Sex Differential in Mortality from All Causes and Ischemic Heart Disease. Am.J. Epidemiol. 1983; 117 (2): 165-72.

WINGARD, D.L. The Sex Differential in Morbidity Rates. Demographic and Behavioral Factors. Am. J. Epidemiol. 1982; 115 (2): 205-16.

WINGARD, D.L. The Sex Differential in Morbidity, Mortality and Lyfestyle. Ann. Rev. Pub. Hlth., 1984; 5: 433-58. (132 ref.) 